\title{
Prioritising outcomes measures for ambulance service care: a three stage consensus methods study
}

\author{
SSM 2013 \\ 57th Annual Scientific Meeting
}

J Coster, J Turner, N Sirwardena, R Wilson, V-H

Phung, A O'Cathain, $F$ Togher, J Nicholl 


\section{Summary}

- Background to the project

- Current ambulance service quality and performance measures

- What do ambulance service stakeholders and PPI representatives think we should measure?

- What is important to patients?

- Delphi study 


\section{Background}

- 4.4 million callers in 2000-01, 9.09 million in 2012-13

- Types of patients and needs changing

- Historically response times measured as a marker of quality

- Difficult to measure
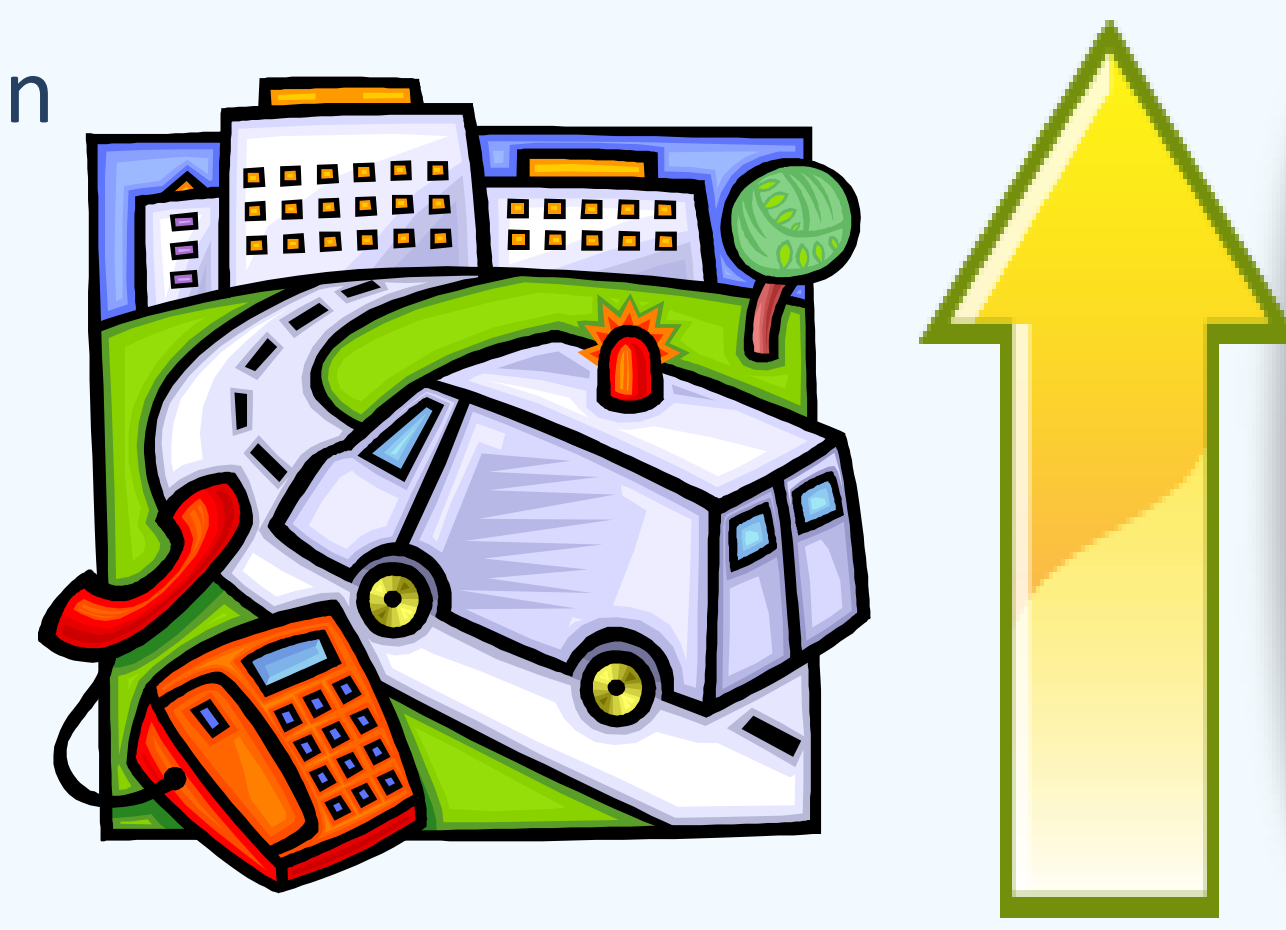
'outcomes' - why? 


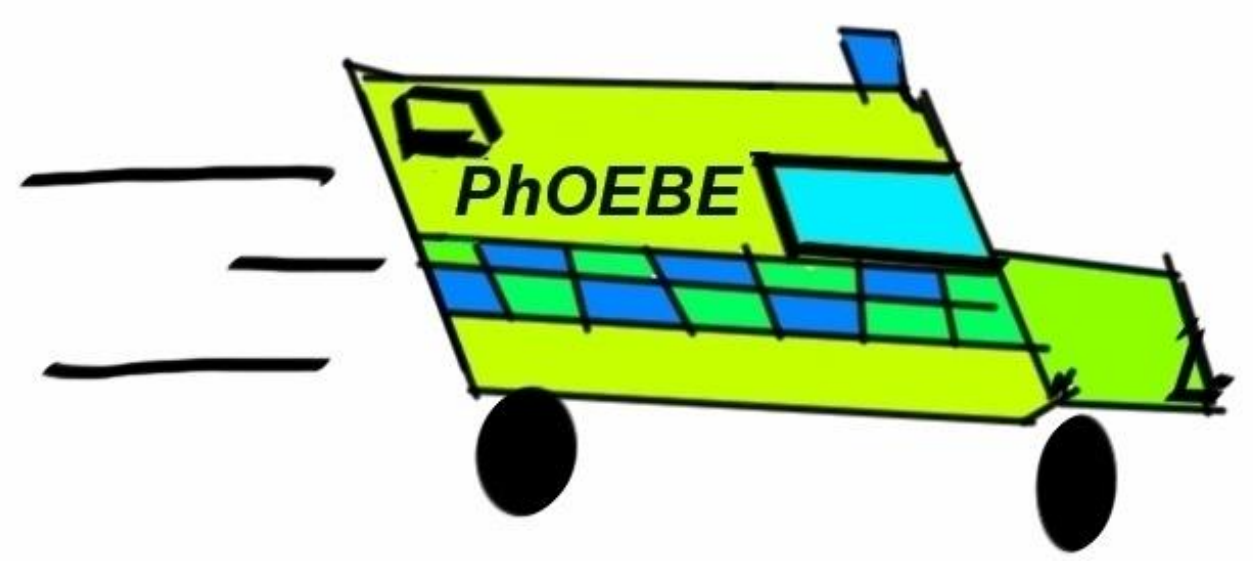

Pre-hospital Outcomes for Evidence Based Evaluation

- 5 year NIHR programme grant

- Develop better ways of measuring the performance, quality and impact of ambulance service care

- Systematic reviews of actual and aspirational outcome measures

- Prioritisation of identified outcomes using consensus event, online survey and Delphi

- Create linked ambulance service/other services data set

- Use outcomes to develop predictive models

- Provide better information about effectiveness and quality of care 


\section{Current ambulance service quality and performance measures}

- Systematic review of current measures

- 405 measures/151 papers

- Patient outcomes 13\%

- Survival and time 60\%

- Why measure time and survival?

- Easy to measure

- Easy to record

- Issues

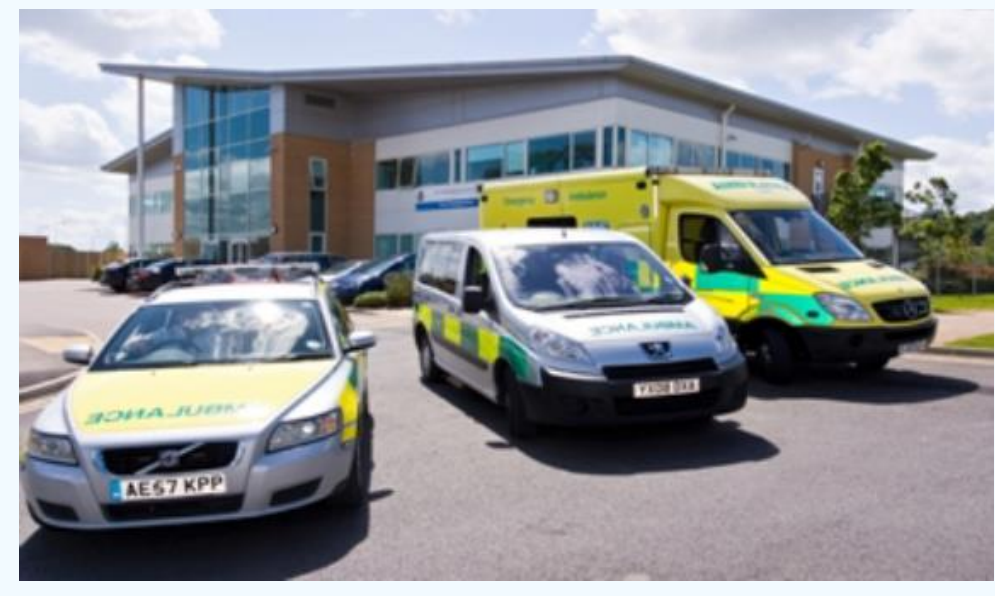

- Only applicable to a small patient group

- No information about quality of care or patient views What about clinical need, patient experience or effectiveness? 


\section{Review of policy documents}

- Current measures

- Response time the predominant measure

- Focus on a few critical conditions

- Patient outcomes: Survival/mortality measures and satisfaction

- Aspirational

- More patient outcome based measures

- Balanced score card approach

- a suite of measures rather than single measures

- Why is it difficult?

- Lack of "joined up" information is a key limiting factor in developing more outcome based measures

- Little effort on developing generic measures that are applicable on a service population rather than condition basis. 


\section{Prioritising outcome measures using consensus methods}

- Large number of time measures - prioritised using an online form

- Consensus event - small group discussion and live vote of key measures and concepts from literature

- Delphi survey

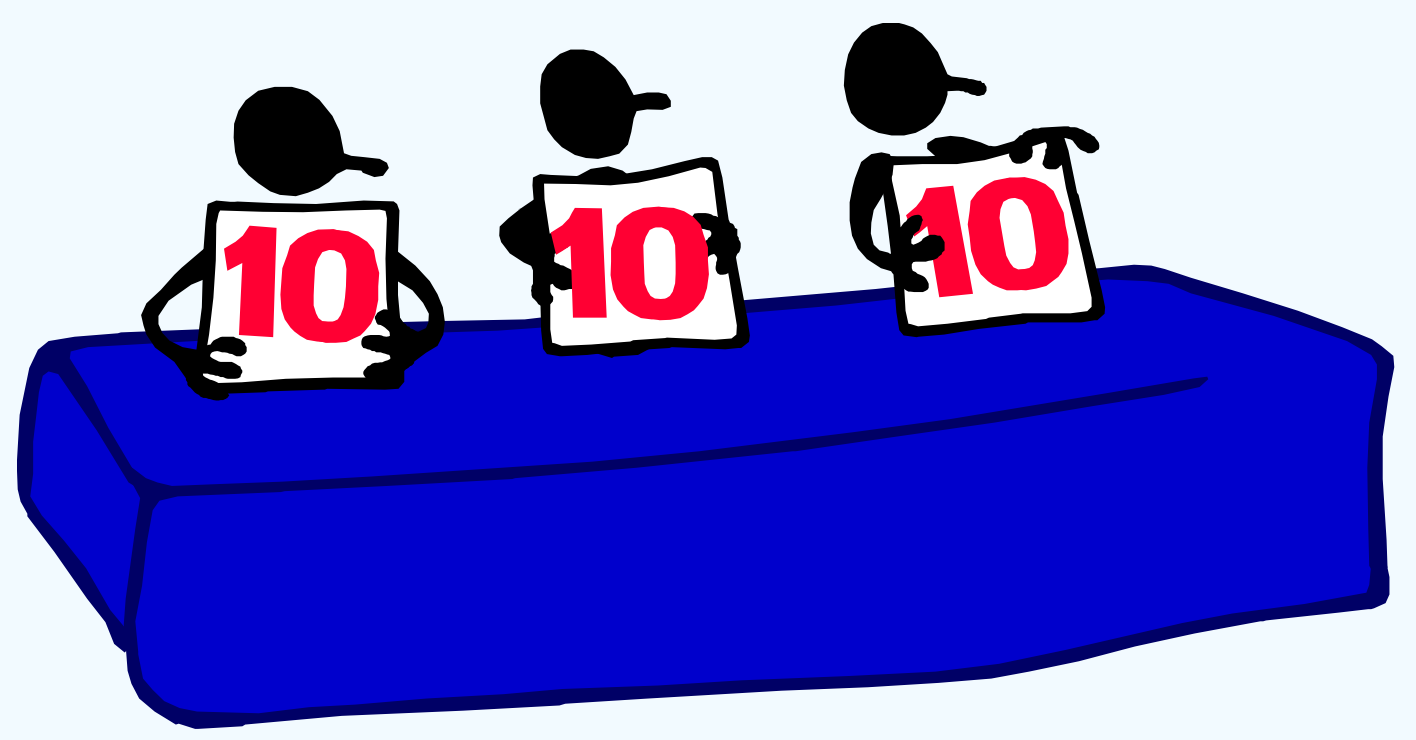




\section{The issue of time}

- Most commonly collected and reported measure

- 23 different time interval measures

- Most common is call to scene

- Recognised as having little relevance or value

- Online survey: which time measures are most useful?

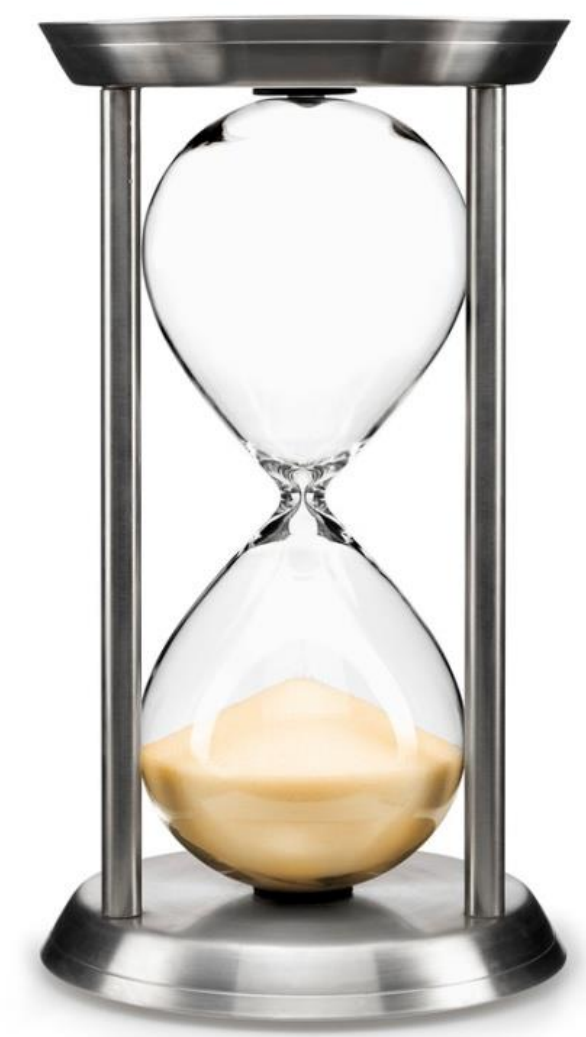




\section{Time measures online survey}

- 28 responses (48\%RR)

- Most important and least important measures
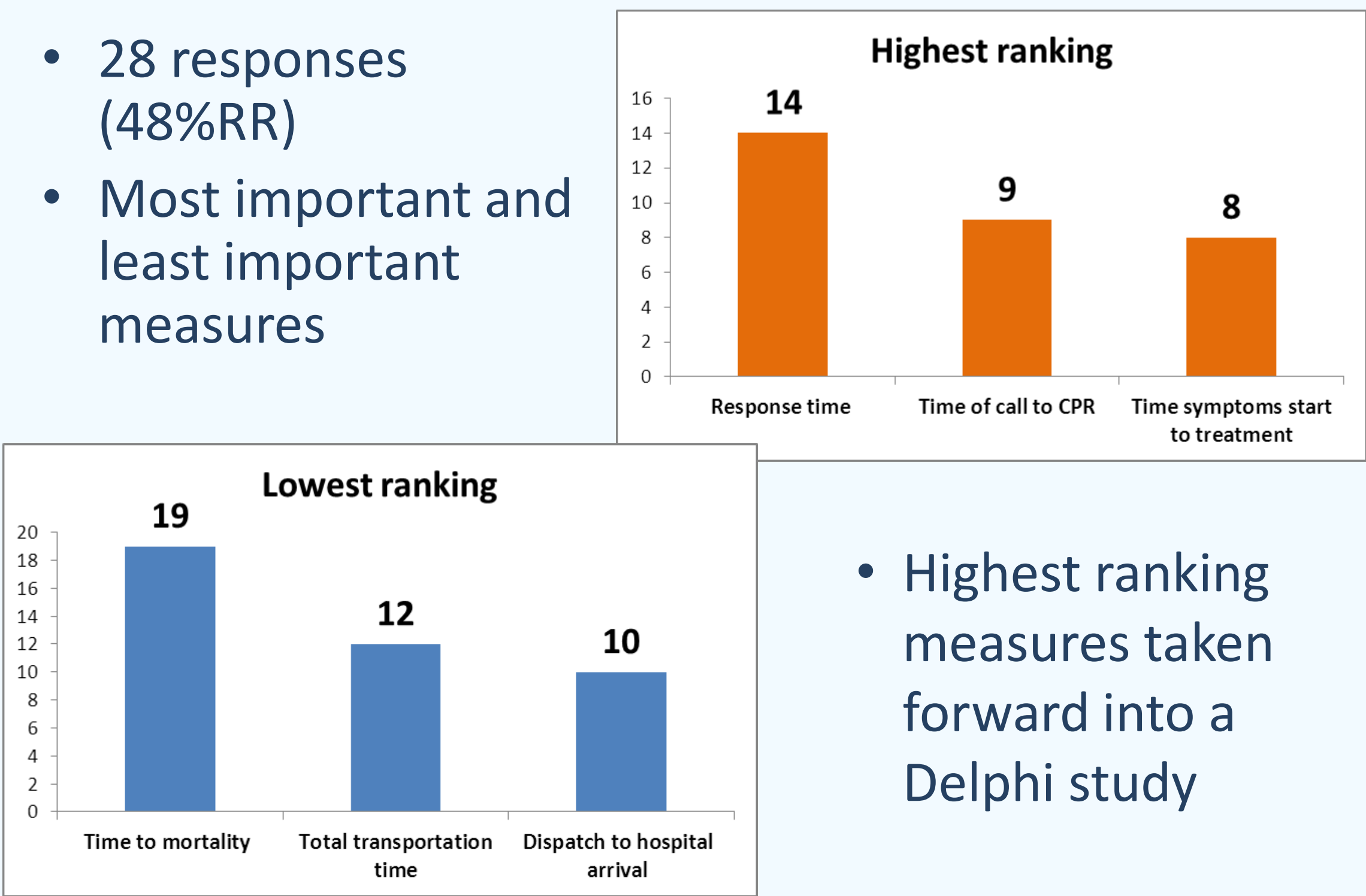

- Highest ranking measures taken forward into a Delphi study 


\section{Consensus Event}

Aim - to prioritise potential measures for measuring ambulance service quality and performance

- 1 day event, small group discussions, live votes

- Ambulance service

- Patient and public

- Commissioners

- Policy makers

- Academic research

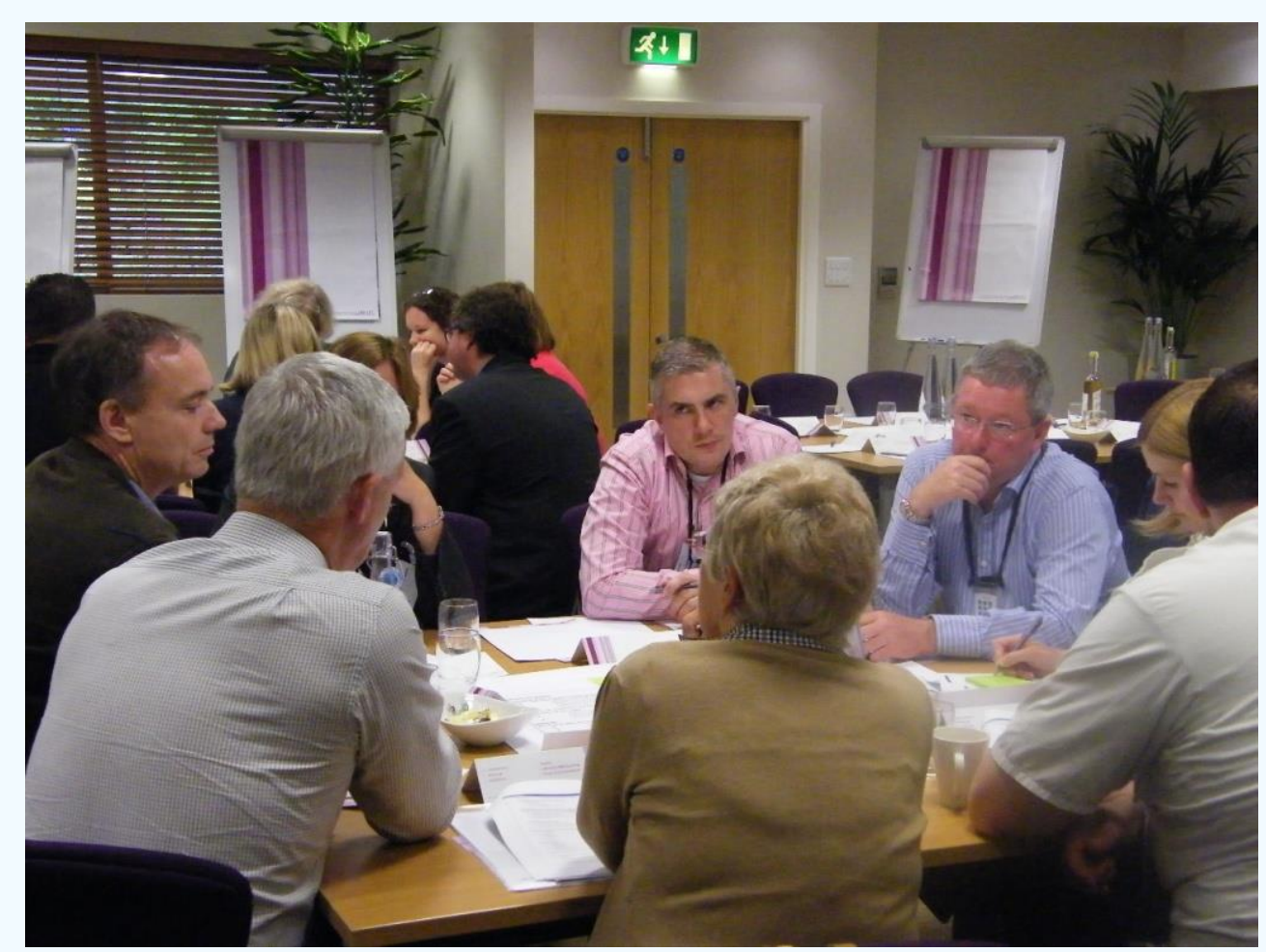




\section{Consensus event results}

\begin{tabular}{|l|l|l|}
\hline Rank & Service/Operational & Essential (n\%) \\
\hline 1 & Completeness and accuracy of patient records & $35(83)$ \\
\hline 2 & Over triage and under triage rates & $31(73)$ \\
\hline 3 & Proportion of calls treated by most appropriate service & $30(71)$ \\
\hline & Patient management & \\
\hline 1 & Accuracy of dispatch decisions & $36(86)$ \\
\hline 3 & $\begin{array}{l}\text { Accuracy of call taker identification of different conditions/ } \\
\text { needs }\end{array}$ & $34(81)$ \\
\hline 3 & Compliance with end of life care plans & $31(74)$ \\
\hline 1 & Patient outcomes & \\
\hline 2 & Pain management and symptom relief & $32(76)$ \\
\hline 3 & Patient experience & $21(50)$ \\
\hline
\end{tabular}




\section{Consensus Event conclusions}

- Accuracy of different types of decision making and compliance with management protocols predominated as essential

- Pain management the most important patient measure

- Management of end of life care was identified by participants

- The electronic voting system which provided instant real time feedback was well received by participants

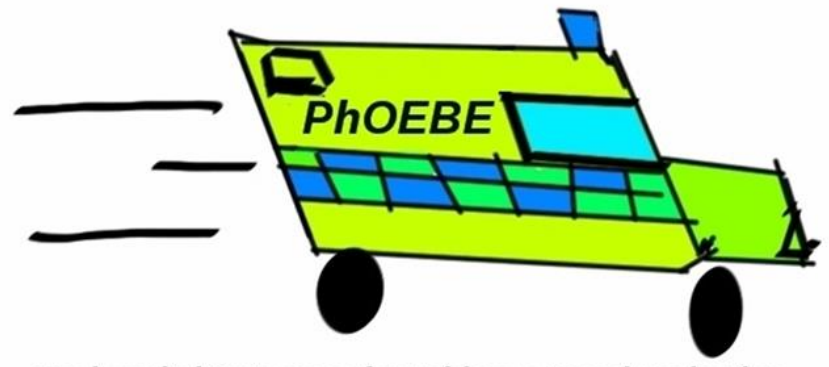




\section{Patient perspective}

- Qualitative interviews for patient experience measures

- 16 interviews with ambulance service users

- Key findings

- Users feel reassured

- Waiting time is acceptable

- Staff who listen and offer clear explanations to users

- Staff who are caring and respectful

- Staff who are thorough

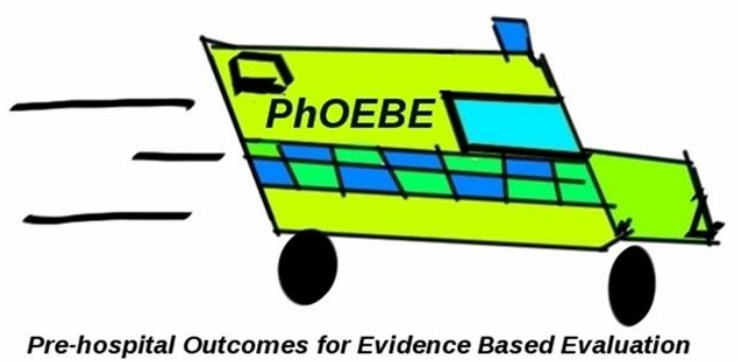




\section{Delphi study development}

- Delphi survey to further refine and prioritise the measures

- Highest ranking measures from the consensus event and time measures online survey

- Also incorporates the findings from patient interviews

- Some high priority concepts difficult to measure or have multiple measures e.g. patient safety, accuracy of dispatch decisions

- Link back to systematic review data to identify measurement methods 


\section{Conclusions}

- Information from multiple sources about potential measures and their



Pre-hospital Outcomes for Evidence Based Evaluation importance

- Key themes: accuracy of processes, compliance

- Patient outcomes: patient experience, pain and patient safety

- Further refined in Delphi study - September 2013 